\title{
Contribution of Microstructural Features at Various Length Scales to the Strength of Additively Manufactured Austenitic Stainless Steels
}

\author{
Joshua D. Sugar ${ }^{1 *}$, Thale R. Smith ${ }^{1}$, and Chris San Marchi ${ }^{1}$ \\ 1. Sandia National Laboratories, Livermore, CA, USA. \\ * Corresponding author: jdsugar@sandia.gov
}

The strength of additively manufactured (AM) steels has been shown to be equivalent to or higher than that of traditionally processed materials $[1,2]$. However, the microstructural features present in AM and conventional steels are substantially different. As one example, Figure 1 shows large-area EBSD maps of (a) directed energy deposited (DED) 304L steel and (b) a 308 e-beam weld. It is clear that the base metal grains in (b) are relatively uniform and equiaxed, while the grains in (a) are elongated and more closely resemble the fusion zone of the weld in (b), but at a much smaller length scale. Despite these microstructural differences, the strength of these materials can be remarkably similar. Therefore, to reproducibly and reliably process steels with AM techniques, a fundamental understanding is needed of how the microstructural features present in AM materials contribute to the overall strength. Coupled with knowledge of how the thermomechanical history affects the uniformity and distribution of these features within a build, one could use this understanding to optimize performance.

Here, we will discuss the measurement of these various microstructural features using microscopy techniques that capture the full range of length scales. Figure 2 shows the features found in these materials including grain boundaries, retained ferrite, solidification cell boundaries, nanoscale oxide particles, and high-density dislocation cells, which cover scales from atomic dimensions (a single dislocation), to tens of microns (typical grain size). Experiments that extract the contribution of each of these features through annealing [3] and build geometry [1] will be discussed. Confidence in AM technology requires coupled microstructure-processing-property relationship experiments like the ones presented here to ultimately enable single builds with reliable performance and without failure [7].

\section{References:}

[1] TR Smith et al., Acta Materialia 164 (2019), p. 728.

[2] YM Wang et al., Nature Materials 17 (2017), p. 63.

[3] TR Smith et al., JOM 70 (2018), p. 358.

[4] M Kamaya, Ultramicroscopy 111 (2011), p. 1189.

[5] C Moussa et al., Ultramicroscopy 179 (2017), p. 63.

[6] C Moussa et al., IOP Conference Series: Materials Science and Engineering 89 (2015), p. 012038.

[7] Sandia National Laboratories is a multimission laboratory managed and operated by National Technology \& Engineering Solutions of Sandia, LLC, a wholly owned subsidiary of Honeywell International Inc., for the U.S. Department of Energy's National Nuclear Security Administration under contract DE-NA0003525. 


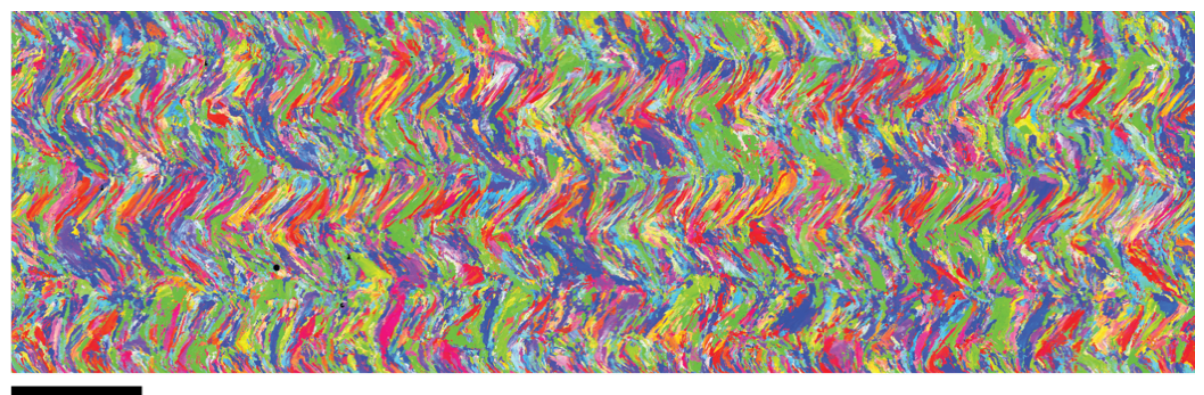

$1 \mathrm{~mm}$

(a)
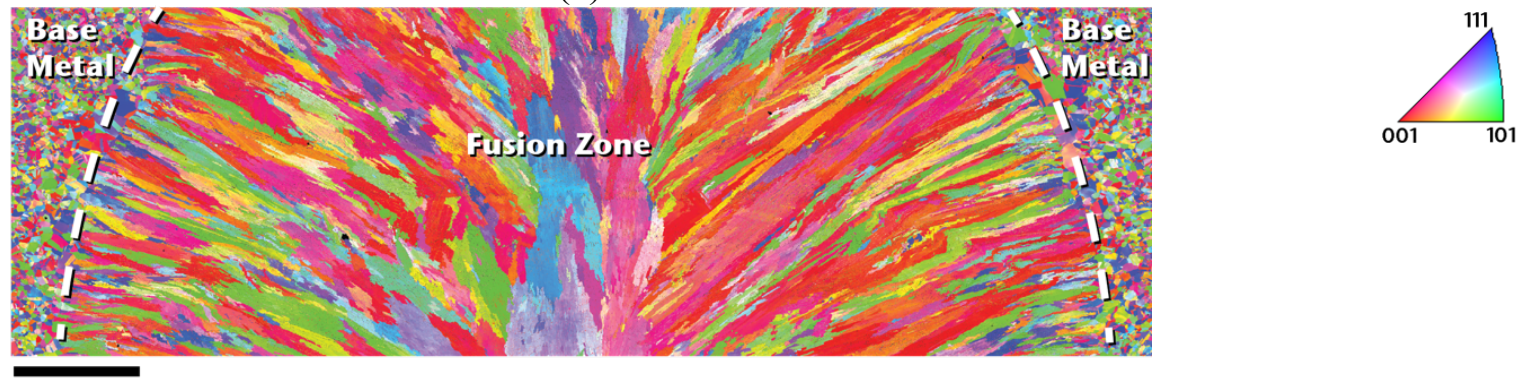

(b)

Figure 1. Large area EBSD IPFZ Maps of (a) Directed Energy LENS deposited 304L stainless steel and (b) conventional 308 stainless steel e-beam weld. These images demonstrate the relevant length scales of the various microstructures and the sensitivity to processing type.

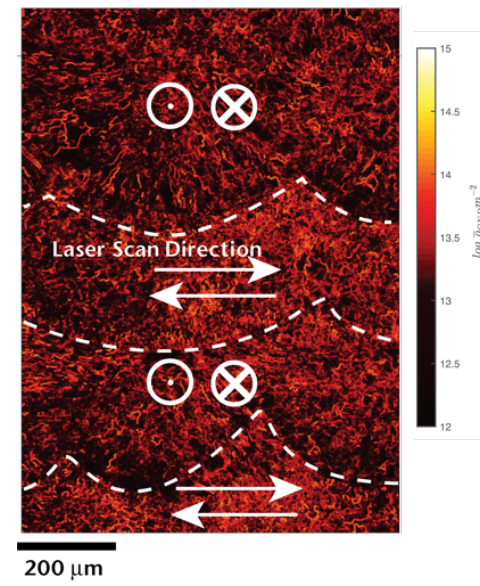

(a)

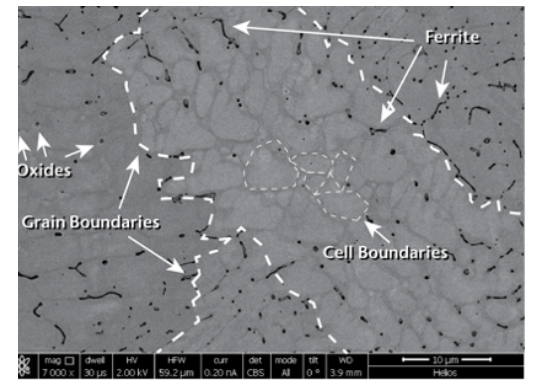

(b)

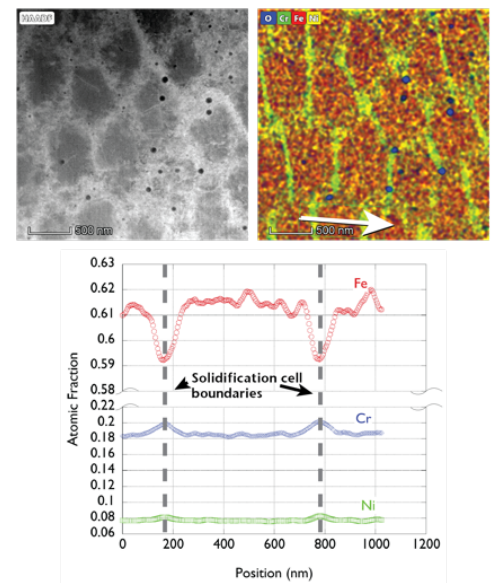

(c)

Figure 2. Images showing microstructural features at various length scales in AM 304L stainless steel. The calculated geometrically necessary dislocation (GND) distribution from EBSD data following [4-6] in (a) shows the distribution of GNDs across several melt pools. The etched and stained micrograph in (b) shows the diverse microstructural features present in directed energy deposited 304L including solidification cells, retained ferrite, grain boundaries, and oxide particles. The TEM micrograph and EDS map and extracted profile in (c) shows the compositional microsegregation that can occur at $100 \mathrm{~s}$ of nanometers in powder bed fusion 304L and its coincidence with the dislocation cells. 